\title{
Flavonoides no controlo sintomático da patologia hemorroidária: uma revisão baseada na evidência
}

Filipe Fontes Alves, ${ }^{1}$ Filipa Martins Guedes, ${ }^{2}$ Joana Oliveira Sousa, ${ }^{3}$ Carolina Macedo Abreu ${ }^{4}$

\section{RESUMO}

Objetivo: A doença hemorroidária é caracterizada pela dilatação dos plexos venosos hemorroidários. Os principais sintomas e sinais são dor, prurido, secreção mucosa, sangramento e desconforto relacionado com o prolapso. Uma das opções terapêuticas são os flavonoides cuja eficácia continua a ser um ponto de debate controverso. Como tal, é objetivo do presente estudo determinar, através de uma revisão baseada na evidência, se a utilização de flavonoides no controlo sintomático é uma opção terapêutica eficaz.

Fontes de dados: National Guideline Clearinghouse, NICE Guidelines Finder, The Cochrane Library, DARE, Bandolier, MEDLINE e referências bibliográficas dos artigos selecionados.

Métodos de revisão: Definição dos critérios de inclusão pelo método PICO: População - Indivíduos com patologia hemorroidária sintomática; Intervenção - Flavonoide; Comparação - Placebo; Outcome - Eficácia do fármaco no controlo sintomático. Pesquisa de normas de orientação clínica, ensaios clínicos aleatorizados, revisões sistemáticas e meta-análises, utilizando os termos MeSH Hemorrhoids e Flavonoids, publicados nos últimos 10 anos em Português, Inglês, Francês e Espanhol. Foi aplicada a escala Strength of Recomendation Taxonomy, da American Academy of Family Physicians, para graduar a evidência e atribuir uma força de recomendação às conclusões.

Resultados: Da pesquisa resultaram 13 artigos, dois dos quais cumpriram os critérios de inclusão, com elevada qualidade científica e dos quais foram traçadas as conclusões, todos eles favoráveis ao uso dos flavonoides, mas com benefício variável de acordo com o sintoma explorado.

Conclusões: A evidência disponível sugere existir benefício na utilização de flavonoides no controlo de sintomas de origem hemorroidária (versus placebo), estando a sua utilização recomendada no controlo da dor e da hemorragia (força de recomendação A). É necessário ter em conta limitações como a subjetividade na avaliação de sintomas por parte dos doentes; a influência da dieta; os flavonoides serem um grupo farmacológico vasto; não existir uma dosagem standard para o tratamento. Estudos adicionais são necessários para esclarecer estas questões.

Palavras-chave: Hemorroidas; Flavonoides.

\section{INTRODUÇÃO}

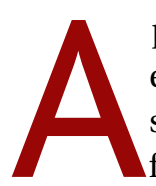

patologia hemorroidária é uma condição de elevada prevalência e estima-se que os seus sintomas afetem $50 \%$ das pessoas em alguma fase da vida. Por esta razão, este é um motivo frequente de recurso aos cuidados de saúde primários. ${ }^{1-2}$ Os sintomas são incomuns antes dos 20 anos, atingindo-se o pico de incidência entre os 45 e os 65 anos. A prevalência parece ser superior em caucasianos e classes socioeconómicas superiores..$^{3-5}$
O plexo venoso hemorroidário é uma estrutura anatómica normal do canal anal e a sua transformação patológica pela alteração da drenagem venosa causa ingurgitamento e estase vascular, associada a dilatação do tecido conectivo. ${ }^{1-2}$ Fatores que propiciam esta transformação incluem agressões repetidas como esforço

1. USF Infesta, ULS de Matosinhos.

2. USF Caravela, ULS de Matosinhos.

3. USF Maresia, ULS de Matosinhos.

4. UCSP São Mamede, ULS de Matosinhos. 
defecatório excessivo, obstipação crónica, dieta pobre em fibras, altas pressões intra-abdominais, gravidez, disfunção do pavimento pélvico, obesidade, influência familiar, entre outros. ${ }^{2-3}$

O ingurgitamento do plexo hemorroidário leva ao aparecimento de sinais e sintomas como prurido, dor, retorragia, sensação de preenchimento da ampola retal com tenesmo defecatório e secreção mucosa com consequente irritação cutânea. Os sintomas podem ser crónicos, surgir em episódios intensos vulgarmente denominados como «crise hemorroidária» ou uma combinação progressiva de ambos. ${ }^{1,6} \mathrm{~A}$ retorragia é o sinal mais comum de apresentação, ${ }^{5}$ mas a dor é o sintoma mais proeminente nos casos de hemorroidas prolapsadas, geralmente quando estranguladas ou trombosadas. ${ }^{1}$ Este sintoma não está tradicionalmente associado a hemorroidas internas e, apesar de poder estar presente, outros problemas devem ser explorados. ${ }^{4}$ Pelo menos $20 \%$ dos doentes que sofrem de patologia hemorroidária apresentam concomitantemente fissuras anais sintomáticas que requerem tratamento adicional para um alívio completo da dor. ${ }^{5} \mathrm{Um}$ exame físico cuidadoso, com inspeção da região anal e toque retal, deve ser realizado a todos os doentes para avaliação da gravidade e descartar outras comorbilidades. ${ }^{1}$ A gravidade da doença é comummente avaliada através de uma escala que guia a escolha entre tratamentos conservadores ou invasivos, focando-se na extensão do prolapso e não no desconforto que os sintomas causam ao doente (Tabela 1).

A abordagem terapêutica das hemorroidas depende da sua localização, grau e da existência de trombo. As intervenções, para além da promoção da educação do doente, devem minimizar os sintomas e prevenir a progressão da doença. ${ }^{2} \mathrm{O}$ tratamento conservador baseiase na modificação da dieta/estilos de vida e uma ingesta adequada de fibras, ${ }^{5-6}$ estando na doença avançada recomendada intervenção cirúrgica. ${ }^{1,6}$

Os flavonoides são um grupo farmacológico heterogéneo, usado maioritariamente no tratamento de insuficiência venosa crónica e hemorroidas. Apesar do seu mecanismo de ação não estar completamente esclarecido, teoriza-se que atuem através do fortalecimento das paredes dos vasos, aumento do tónus venoso, facilitação da drenagem linfática e normalização da permeabilidade capilar. ${ }^{5}$ Apesar de existirem múltiplos

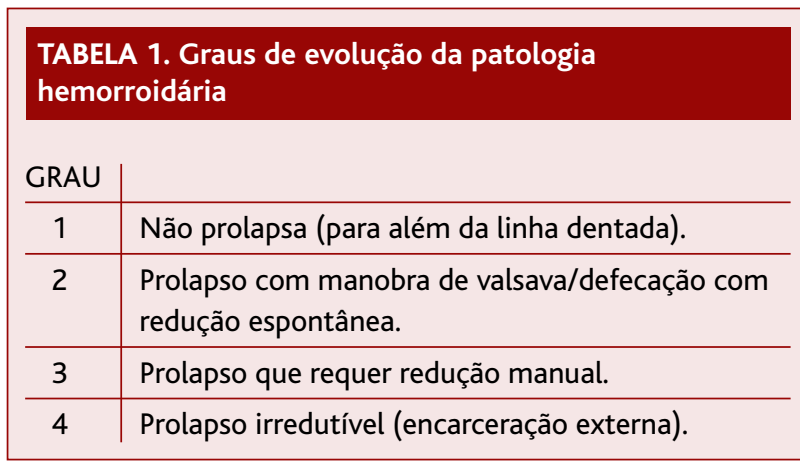

estudos sobre a aplicação de flavonoides no controlo sintomático desta doença, este continua a ser um ponto de debate controverso entre os médicos, com profissionais a relatarem sucesso com a sua utilização e outros mantendo-se céticos, coexistindo atitudes terapêuticas inconsistentes. ${ }^{5}$

O objetivo dam presente revisão baseada na evidência (RBE) é determinar a eficácia dos flavonoides no controlo sintomático na patologia hemorroidária.

\section{MÉTODOS}

Recorrendo às bases de dados National Guideline Clearinghouse, NICE Guidelines Finder, The Cochrane Library, DARE, Bandolier e MEDLINE foi realizada a pesquisa de normas de orientação clínica, meta-analises, ensaios clínicos aleatorizados e revisões sistemáticas. A pesquisa incluiu todas as publicações em Português, Inglês, Espanhol e Francês, de estudos em humanos, com um máximo de 10 anos de publicação (relativamente à data de pesquisa: 28/05/2018). Foi utilizada uma combinação dos termos MeSH Hemorrhoids e Flavonoids.

Os critérios de inclusão dos artigos foram definidos de acordo com o modelo PICO: ${ }^{7}$ População - Portadores de patologia hemorroidária; Intervenção - Utilização de flavonoides no controlo sintomático da doença hemorroidária; Controlo - Placebo; Outcomes - Redução de sintomas e sinais como dor, prurido, sangramento (retorragia) e edema/sensação de tumefação.

Definiu-se, como critérios de exclusão, grávidas, doentes submetidos a hemorroidectomia e/ou a outras intervenções invasivas. Foram excluídos igualmente todos os artigos duplicados ou incluídos em revisões sistemáticas e meta-análises recentes. Os 


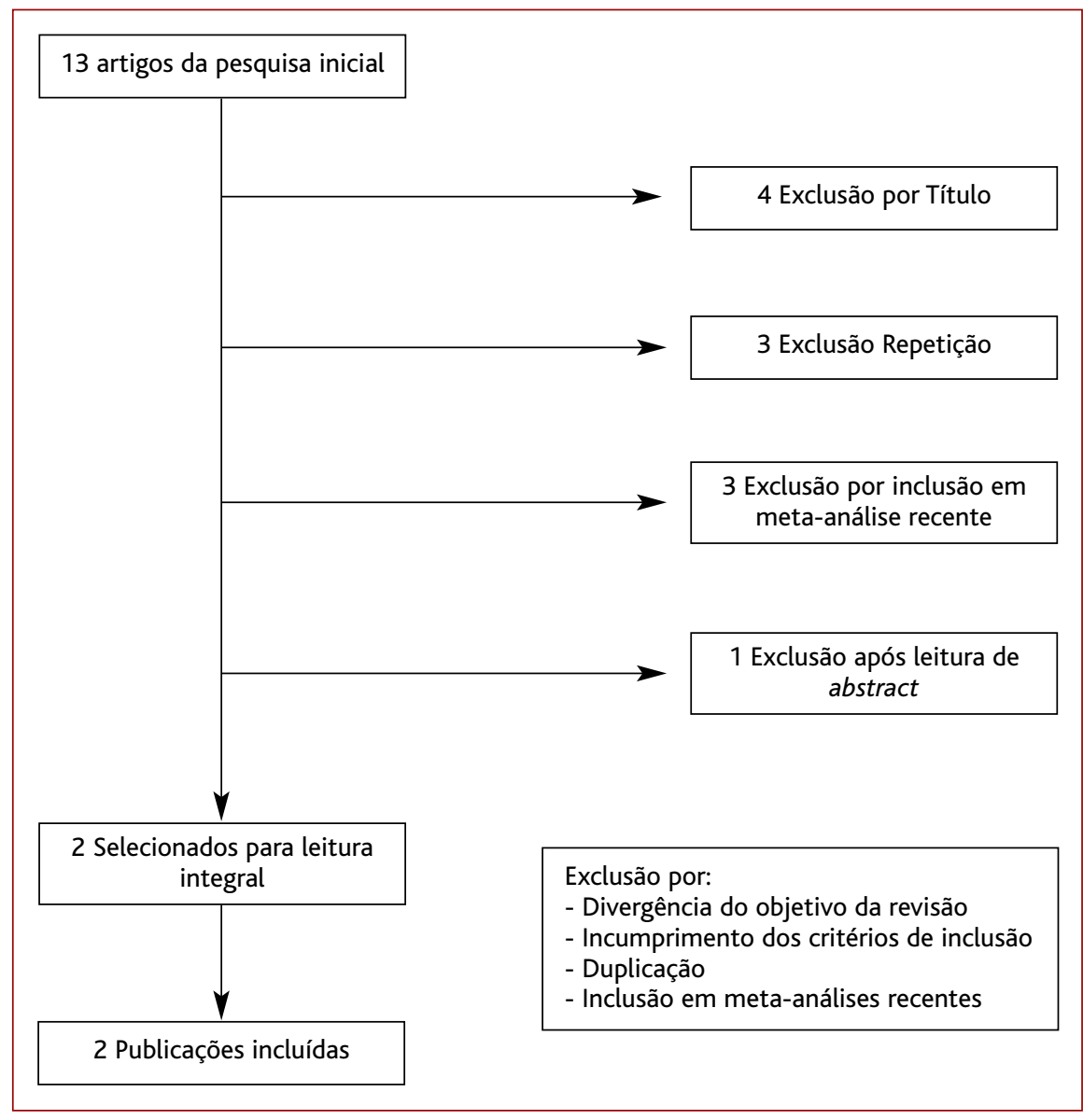

Figura 1. Seleção de artigos.

resultados da pesquisa foram submetidos a avaliação da qualidade dos resultados e da sua força de recomendação utilizando a escala SORT (Strength of Recommendation Taxonomy), da American Academy of Family Physicians. ${ }^{8}$

\section{RESULTADOS}

Da pesquisa resultaram 13 artigos. Por divergirem do objetivo desta revisão e não cumprirem os critérios de inclusão, quatro foram excluídos após leitura do título, três artigos por duplicação, três por terem sido incluídos em revisões mais recentes (meta-análise Cochrane publicada em 2012) e um após leitura do abstract. Desta seleção resultaram duas publicações: um ensaio clínico controlado aleatorizado e uma meta-análise (Figura 1).

O ensaio clínico aleatorizado controlado incluído nesta RBE foi realizado em Itália por Giannini ${ }^{6}$ e publi- cado em 2015 na revista Techniques in Coloproctology. Relata um estudo multicêntrico, no qual se comparou a administração oral de flavonoides a placebo no tratamento da crise hemorroidária, tendo sido avaliados os seus efeitos na dor, sangramento, prurido, necessidade de recorrer a analgesia e prolapso. A intensidade dos sintomas foi reportada pelos doentes utilizando uma escala visual analógica de dez itens. A coorte de 134 doentes foi separada de forma aleatória em dois grupos, um a receber saquetas com uma combinação de diosmina (300mg), hisperidina (300mg) e troxerrutina (300mg) e o outro a receber saquetas idênticas com placebo. O processo de aleatorização, de administração do tratamento e de análise estatística foi exposto de forma clara e a tripla ocultação foi conseguida. Foi administrada uma saqueta do preparado três vezes por dia durante tês dias, seguido de duas vezes por dia durante dois dias, uma saqueta diária nos sete dias seguintes e ao $13^{\circ}$ dia passaram a comprimido oral diário até completar 42 dias de tratamento. Os comprimidos apresentam uma composição semelhante, com exceção da dose de hisperidina que é menor (100mg). O follow-up foi completo, sem abandonos precoces ou exclusões do estudo. Segundo os resultados obtidos foi conseguida melhoria sintomática mais precoce no grupo a receber tratamento ativo (com significância estatística) no que toca a redução da dor $(p<0,0001)$, sangramento $(p=0,015)$, prurido $(p=0,006)$ e edema $(p=0,0016$; apenas com uso continuado) e com menor necessidade de analgesia oral $(p=0,003)$. Não foi encontrada uma diferença estatisticamente significativa no que toca à ocorrência de prolapso $(p=0,09)$. Ambos os grupos mostraram que ao longo do tempo os sintomas tendem a desvanecer; no entanto, foi evidente que o grupo a receber 


\begin{tabular}{|c|c|c|}
\hline ENSAIOS CLÍNICOS & Z. Jiang (2006) ${ }^{9}$ & I. Giannini $(2015)^{6}$ \\
\hline $\begin{array}{l}\text { Tipo de estudo e } \\
\text { população }\end{array}$ & $\begin{array}{l}\text { Ensaio aleatorizado controlado; double-blind; } \\
N=90 \\
\text { Doentes entre os } 18 \text { e os } 75 \text { anos de idade, não } \\
\text { gestantes, com patologia hemorroidária } \\
\text { sintomática, sem intervenção prévia }\end{array}$ & $\begin{array}{l}\text { Ensaio aleatorizado controlado; triple-blind; } \\
N=134 \\
\text { Doentes entre os } 18 \text { e os } 75 \text { anos de idade, não } \\
\text { gestantes, com patologia hemorroidária } \\
\text { sintomática, sem intervenção prévia }\end{array}$ \\
\hline Fase da doença & \multicolumn{2}{|c|}{ aguda («Crise hemorroidária») } \\
\hline $\begin{array}{l}\text { Tratamento em } \\
\text { comparação com } \\
\text { placebo }\end{array}$ & $\begin{array}{l}\text { MPFF } 500 \mathrm{mg} \text { ( } 90 \% \text { diosmina, } 10 \% \text { hisperidina); } 6 \text { id } \\
\text { por } 4 \text { dias, seguido de } 4 \text { id por } 3 \text { dias; }\end{array}$ & $\begin{array}{l}\text { Preparado de flavonoides } 900 \mathrm{mg} \text { (diosmina } \\
300 \mathrm{mg} \text {, hisperidina } 300 \mathrm{mg} \text { e troxerrutina } 300 \mathrm{mg} \text { ); } \\
\text { 3id por } 3 \text { dias, seguido de } 2 \text { id por } 2 \text { dias, seguido de } \\
\text { 1id por } 7 \text { dias; }\end{array}$ \\
\hline \multicolumn{3}{|l|}{ ENDPOINTS } \\
\hline dor & BS $(p<0,001)$ & BS $(p<0,001)$ \\
\hline sangramento & BS $(p=0,047)$ & BS $(p=0,015)$ \\
\hline prurido & NS & BS ( $p=0,006$; apenas com utilização continuada) \\
\hline edema & BS $(p<0,001)$ & BS ( $p=0,0016$; apenas com utilização continuada) \\
\hline secreção mucosa & NS & Sem dados \\
\hline prolapso & NS & NS \\
\hline $\begin{array}{l}\text { Nível da qualidade } \\
\text { da evidência }{ }^{7}\end{array}$ & 1 & 1 \\
\hline
\end{tabular}

Legenda: BS = Benefício estatisticamente significativo; NS = Benefício não estatisticamente significativo; MPFF = Micronised purified flavonoid fraction.

tratamento ativo ficou assintomático mais precocemente e com resultados que foram favorecidos pelo tratamento continuado, especialmente no que se refere à ocorrência de sangramento (Tabela 2).

Por este ser um estudo controlado, aleatorizado, com ocultação tripla, cuja intenção é tratar, com endpoints orientados para o doente, com um número de participantes adequado (poder amostral $>80 \%$ ) e por apresentar um follow-up $>80 \%$, atribuiu-se à qualidade da evidência o nível um, de acordo com a escala SORT. ${ }^{8}$

A meta-análise obtida através da pesquisa é uma revisão Cochrane publicada em 2012. ${ }^{5}$ Os autores tiveram por objetivo determinar a eficácia e segurança da utilização de venotrópicos no controlo sintomático das hemorroidas, assim como determinar os seus efeitos pós-hemorroidectomia. Por todos os venotrópicos utilizados serem flavonoides, este será o termo empregue. Foram incluídos nesta revisão 24 artigos, três que comparam diferentes flavonoides, um que comparava flavonoides versus intervenções invasivas e os restantes 20 versus placebo. Quatro dos casos controlo fizeram avaliação sintomática pós-hemorroidectomia. A maioria (20) foram realizados em países de rendimento elevado, sendo que praticamente todos foram desenvolvidos na Europa (14) e Ásia (9), regiões onde esta terapêutica é aplicada com maior frequência. A maioria dos estudos incluídos nesta revisão incluíram adultos de ambos os sexos, com exceção de um estudo em que foram apenas incluídas grávidas.

Apesar de alguns destes estudos não irem de encontro à questão PICO desta RBE, como por exemplo aqueles que incluem grávidas, avaliação pós-hemorroidectomia e estudos comparativos versus placebo, a metaanálise reporta uma investigação estatística detalhada com discussão por subgrupos que são relevantes. Por este motivo, optou-se por apresentar apenas os dados 


\begin{tabular}{|c|c|c|}
\hline $\begin{array}{l}\text { Outcomes } \\
\text { ( } \mathrm{n}^{\circ} \text { artigos data-pooled; } \\
\text { população) }\end{array}$ & Intervenção & Resultados \\
\hline $\begin{array}{l}\text { Dor } \\
\text { (2 artigos) }\end{array}$ & $\begin{array}{l}\text { MPFF } 1 \mathrm{~g} 1 \text { tid } 4 d \text { seguido de bid } 3 d \\
\text { MPFF 500mg 6id 4d seguido de qid } 3 d\end{array}$ & $\begin{array}{l}\text { Benéfico } \\
\text { Não estatisticamente significativo no alívio da } \\
\text { dor }(\mathrm{OR}=0,11 ; \mathrm{IC} 95 \%, 0,01-1,11 ; p=0,06) \\
\text { Estatisticamente heterogéneo }\left(I^{2}=85 \%\right)\end{array}$ \\
\hline $\begin{array}{l}\text { Hemorragia } \\
\text { ( } 2 \text { artigos) }\end{array}$ & $\begin{array}{l}\text { MPFF 500mg 6id 4d seguido de qid } 3 d \\
\text { MPFF 500mg 6id } 4 d \text { seguido de qid } 3 d\end{array}$ & $\begin{array}{l}\text { Benéfico } \\
\text { Estatisticamente significativo (OR=0,12; IC95\%, } \\
0,04-0,37 ; P=0,0002) \\
\text { Sem heterogeneidade estatística }\left(I^{2}=0 \%\right)\end{array}$ \\
\hline $\begin{array}{l}\text { Secreção mucosa } \\
\text { ( } 2 \text { artigos) }\end{array}$ & $\begin{array}{l}\text { MPFF } 1 g \text { tid } 4 d \text { seguido de bid } 3 d \\
\text { MPFF 500mg 6id } 4 d \text { seguido de qid } 3 d\end{array}$ & $\begin{array}{l}\text { Benéfico } \\
\text { Estatisticamente significativo (OR=0,12; IC95\%, } \\
0,04-0,42 ; p=0,0008) \\
\text { Sem heterogeneidade estatística }\left(I^{2}=0 \%\right)\end{array}$ \\
\hline $\begin{array}{l}\text { Prurido } \\
\text { (1 artigo) }\end{array}$ & MPFF 500mg 6id 4d seguido de qid 3d & $\begin{array}{l}\text { Benéfico } \\
\text { Não estatisticamente significativo }(O R=0,16 \text {; } \\
\text { IC95\%, } 0,07-0,079 ; p=0,02)\end{array}$ \\
\hline $\begin{array}{l}\text { Melhoria geral } \\
(4 \text { artigos; } 145+126)\end{array}$ & $\begin{array}{l}\text { Oxerrutina } 2 \mathrm{~g} \text { bid } \\
\text { MPFF } 500 \mathrm{mg} 3 \text { bid } 7 \mathrm{~d} \text { seguido de } 2 \text { bid } 3 \mathrm{~d} \\
\text { Dobesilato de cálcio } 500 \mathrm{mg} \text { tid } 7 \mathrm{~d} \text { seguido de } \\
\text { bid } 7 \text { dias } \\
\text { MPFF } 2 \mathrm{~g} 4 \text { tid } 4 \mathrm{~d} \text { seguido de } 1 \mathrm{~g} 2 \text { bid } 10 \mathrm{~d}\end{array}$ & $\begin{array}{l}\text { Benéfico } \\
\text { Estatisticamente significativo (OR=9,6; IC95\%, } \\
3,7957-24,41 ; p<0,00001) \\
\text { Sem heterogeneidade estatística }\left(I^{2}=0 \%\right)\end{array}$ \\
\hline
\end{tabular}

Legenda: MPFF = Micronized purified flavonoid fraction (90\% diosmina, 10\% hisperidina). Número de tomas diárias: bid=2, tid=3, qid=4, 6id=6; ${ }^{*} \mathrm{~d}=$ número de dias a tomar.

pertinentes à nossa questão PICO e individualizar certos aspetos necessários de serem particularizados. Os autores da meta-análise, apesar de considerarem os resultados de todos os estudos, na análise estatística apenas combinaram pools de doentes dos ensaios de maior qualidade metodológica, que relatam de forma detalhada e completa os seus dados e que apresentam formatação semelhante dos seus elementos. Destes estudos traçaram as suas principais conclusões.

Os outcomes explorados, relevantes para a presente RBE, são dor, prurido, sangramento, secreção mucosa e melhoria sintomática geral (Tabela 3 ).

\section{Dor}

Pela análise de dois estudos, com um total de 190 participantes, os autores declaram que a utilização de flavonoides está associada a benefício no controlo álgico, mas sem significância estatística (OR=0,11; IC95\%, $0,01-1,11 ; p=0,06)$ e com grande heterogeneidade entre os resultados $\left(\mathrm{I}^{2}=85 \%\right)$. Ambos os estudos apresentam adequada qualidade metodológica, mas diferentes posologias e formulações foram utilizadas. Cospite (1994) recorreu a diosmina como tratamento de crise hemorroidária com menos de três dias de evolução, tendo sido administrados um grama três vezes por dia durante quatro dias, seguidos de duas vezes por dia nos três dias seguintes, com resultados superiores no controlo da dor quando comparado ao estudo de Jiang (2006), que optou por micronized purified flavonoid fraction ( $90 \%$ diosmina, $10 \%$ hisperidina) no tratamento de crise com menos de $48 \mathrm{~h}$ de evolução, 500mg seis vezes por dia durante quatro dias seguidos, de três 
vezes por dia nos três dias seguintes. Pela heterogeneidade dos resultados e por Cospite não ter declarado de forma óbvia o método de ocultação, os autores da metaanálise atribuíram à qualidade da evidência um grau de moderado, de acordo com a escala GRADE. ${ }^{9}$

\section{Sangramento}

Pela análise de dois estudos, com um total de 190 participantes, os autores declaram que a utilização de flavonoides está associada a benefício estatisticamente significativo na diminuição do sangramento (OR=0,12; IC95\%, $0,04-0,37 ; p=0,0002)$, sem heterogeneidade estatística entre estudos $\left(\mathrm{I}^{2}=0 \%\right)$. Os dois estudos avaliaram o tratamento em fases distintas da doença (patologia crónica e crise hemorroidária), mas o outcome referente à ausência de sangramento ao quarto dia de tratamento foi idêntico. Apesar dos estudos não apresentarem grandes limitações metodológicas, Misra (2000) não foi claro ao declarar o método de ocultação e, por isto, os autores da meta-analise atribuem à qualidade da evidência um grau de moderado, de acordo com a escala GRADE. ${ }^{9}$

\section{Prurido}

Através da análise de dois estudos, com um total de 176 participantes, os autores declaram que a utilização de flavonoides está associada a benefício estatisticamente significativo no controlo do prurido ( $\mathrm{OR}=0,23$; IC95\%, 0,07-0,79; $p=0,02$ ), sem heterogeneidade estatística entre estudos $\left(I^{2}=0 \%\right)$. Pela elevada qualidade metodológica dos estudos, os autores atribuem à qualidade da evidência um grau elevado, segundo a escala GRADE. ${ }^{9}$ No entanto, por motivo não especificado, para este outcome foram agrupados estudos com intervenções distintas (intervenção exclusivamente médica [Jiang (2006) ${ }^{10}$ versus intervenção médica pós-hemorroidectomia: Aba-bai-ke-re (2011)]. O estudo de Jiang demonstra benefício com a utilização de flavonoides, mas sem significância estatística $(p=0,24)$, com um intervalo de confiança amplo (OR=0,16; IC95\%, 0,07$-0,79$ ). Atendendo apenas às conclusões deste estudo, os flavonoides apresentam um benefício não significativo no controlo do prurido de origem hemorroidária (sem intervenção invasiva prévia).

\section{Secreção mucosa}

Através da análise de dois estudos, com um total de
139 participantes, os autores declaram que existe benefício estatisticamente significativo no controlo da passagem involuntária de secreções mucosas $(\mathrm{OR}=0,12$; IC95\%, 0,04-0,42; $p=0,0008)$, sem heterogeneidade entre estudos $\left(\mathrm{I}^{2}=0 \%\right)$. Apesar da adequada qualidade metodológica, um dos estudos não declara de forma explícita os critérios de ocultação e, por isso, os autores atribuíram à qualidade da evidência um grau de moderado, de acordo com a escala GRADE. ${ }^{9}$

\section{Melhora sintomática geral}

Através da análise de cinco estudos, com um total de 368 participantes, os autores declaram que existe uma melhoria sintomática geral reportada pelos doentes que é estatisticamente significativa ( $\mathrm{OR}=15,99$; IC95\%, 5,97$-42,84 ; p<0,00001)$, mas com alguma heterogeneidade entre os resultados $\left(I^{2}=39 \%\right)$. No entanto, esta avaliação incorporou o único estudo (desta meta-análise) que incluiu grávidas. Realizando a sua exclusão, pela análise restrita dos restantes quatro estudos, com um total de 271 participantes, obteve-se melhoria geral estatisticamente significativa (OR=9,63; IC95\%, 3,8-24,41; $p<0,00001$ ), mas sem heterogeneidade entre resultados $\left(\mathrm{I}^{2}=0 \%\right)$, o que demonstra que a consistência dos resultados para este subgrupo é superior à reportada.

A maioria dos dados aponta para benefício no controlo sintomático das hemorroidas, com a maioria dos outcomes apresentando significância estatística. Existe benefício comprovado no tratamento e controlo dos sintomas hemorroidários e, por este motivo, os autores da meta-análise Cochrane recomendam a utilização de flavonoides no seu tratamento. Às conclusões desta meta-análise atribuiu-se um nível de evidência dois, de acordo com a escala SORT. ${ }^{8}$

É necessário considerar que várias limitações foram encontradas. Primeiro, a subjetividade dos sintomas por si só é limitante na avaliação e múltiplas escalas distintas foram empregues pelos diversos estudos, sujeitas a interpretações pessoais tanto por parte dos doentes como dos investigadores. No entanto, também foram utilizadas escalas dicotómicas, mais objetivas, pois avaliaram apenas se o sintoma estava presente ou não (o que também representa uma limitação à avaliação do percurso da doença). Apesar de em todos os casos controlo terem sido feitas recomendações dietéticas e de estilo de vida aos participantes, e tendo 
conhecimento de que este fator é dos que maior impacto tem na doença hemorroidária ${ }^{4}$ não foi possível seguir de forma rigorosa nem de assegurar a compliance dos doentes. A própria localização geográfica da realização do estudo poderá ter impacto, com países onde a dieta é culturalmente mais rica em fibras a ter resultados díspares daqueles onde a dieta é mais obstipante. Os flavonoides representam múltiplas moléculas e formulações. Na grande diversidade de moléculas, posologias e dosagens utilizadas nos vários estudos encontrou-se uma justificação simples para a grande heterogeneidade encontrada nos resultados.

Idealmente mais estudos de qualidade seriam necessários para elevar a confiança das conclusões e os próprios autores advertem que não podem ser extrapoladas para doentes com hemorroidas trombosadas, graus mais avançados da doença e outros subgrupos de doentes.

Contudo, um dos estudos avaliados nesta meta-análise destaca-se pela sua qualidade metodológica, Jiang $(2006)^{10}$ (Tabela 2), realizado na China e publicado na Current Medical Research and Opinion. Contou com total de 90 doentes com crise hemorroidária com duração inferior a 48 horas, cujo tratamento (descrito anteriormente) foi comparado a placebo. Os doentes foram avaliados no primeiro, quarto e sétimo dia, no que toca a dor, sangramento, prurido, edema e prolapso. Os sintomas foram reportados pelos doentes e os sinais pelos médicos investigadores e quantificados, utilizando uma escala de quatro pontos (resolução, melhoria significativa, melhoria minor, sem efeito). Foi obtido benefício estatisticamente significativo com flavonoides no que toca a controlo da dor $(p<0,0001)$, sangramento $(p=0,047)$ e edema ( $p<0,001$; não significativo a longo prazo). Os restantes outcomes, prurido e prolapso, não obtiveram um benefício estatisticamente significativo. Por se tratar de um estudo controlado, aleatorizado, double-blind, com um follow-up de $100 \%$, de boa qualidade, atribuímos o nível um da escala SORT à qualidade da evidência.

\section{CONCLUSÕES}

Os estudos incluídos nesta revisão apontam para uma recomendação convergente: benefício na utilização de flavonoides no tratamento sintomático das hemorroidas em adultos, sem hemorroidectomia prévia, em populações não grávidas. Por estas conclusões serem traçadas com base em estudos de boa qualidade científica, os autores desta revisão atribuem uma força de Recomendação A (escala SORT). Considera-se necessário esclarecer que pela questão PICO ser abrangente, incluindo melhoria de qualquer sintoma, esta força de recomendação aplica-se principalmente a controlo da dor e da hemorragia, as únicas com benefício significativo e concordante em vários estudos de elevada qualidade científica Tabela 2). À sua utilização para controlo dos restantes sintomas, prurido e secreção mucosa, atribuiu-se uma força de recomendação B pelas conclusões serem obtidas de estudos com fragilidades metodológicas que levam a algum risco de viés. A recomendação «A» apenas poderá ser feita à crise hemorroidária, pois foi essa a fase da doença que os estudos abordaram. Apesar de existir evidência de redução de edema não foi demonstrado que flavonoides tenham impacto na redução do prolapso e sintomas associados.

Um dos interesses desta revisão é mitigar a controvérsia envolvendo a indicação terapêutica deste grupo farmacológico. Este tema continua a ser polémico, com atitudes terapêuticas divergentes entre médicos que lidam com este problema de saúde. Por um lado, criamse expectativas irrealistas sobre certos tratamentos e, por outro, privam-se doentes de opções terapêuticas. A própria informação que é cedida aos doentes sobre a eficácia dos flavonoides não é uniforme e tais discordâncias geram desentendimentos entre profissionais e quebra da confiança que o doente deposita nos seus médicos.

Os estudos são unânimes quanto ao benefício dos flavonoides no controlo sintomático da doença hemorroidária (versus placebo). No entanto, a relevância clínica deste benefício não é idêntica para todos os sintomas e os médicos devem ter isso em conta. Igualmente importante é reconhecer que para se conseguir a individualização do tratamento a cada doente e poder atuar em determinados subgrupos específicos são necessários mais estudos de grande qualidade metodológica, com dosagens e fármacos equivalentes (pois uma posologia standard não pôde ser inferida), idealmente na população portuguesa.

\section{REFERÊNCIAS BIBLIOGRÁFICAS}

1. Davies $D$, Bailey J. Diagnosis and management of anorectal disorders in the primary aare setting. Prim Care. 2017;44(4):709-20. 
2. Fernandes V, Camacho A. Doença hemorroidária. Rev Port Coloproct. 2009;6(2):36-43.

3. Mott T, Latimer K, Edwards C. Hemorrhoids: diagnosis and treatment options. Am Fam Physician. 2018;97(3):172-9.

4. Guttenplan M. The evaluation and office management of hemorrhoids for the gastroenterologist. Curr Gastroenterol Rep. 2017;19(7):30.

5. Perera N, Liolitsa D, lype S, Croxford A, Yassin M, Lang P, et al. Phlebotonics for haemorrhoids. Cochrane Database Syst Rev. 2012;(8): CD004322.

6. Giannini I, Amato A, Basso L, Tricomi N, Marranci M, Pecorella G, et al. Flavonoids mixture (diosmin, troxerutin, hesperidin) in the treatment of acute hemorrhoidal disease: a prospective, randomized, triple-blind, controlled trial. Tech Coloproctol. 2015;19(6):339-45.

7. Akobeng AK. Principles of evidence based medicine. Arch Dis Child. 2005;90(8):837-40.

8. Ebell MH, Siwek J, Weiss BD, Woolf SH, Susman J, Ewigman B, et al. Strength of Recommendation Taxonomy (SORT): a patient-centered approach to grading evidence in the medical literature. J Am Board Fam Pract. 2004;17(1):59-67.
9. Atkins D, Best D, Briss PA, Eccles M, Falck-Ytter Y, Flottorp S, et al. Grading quality of evidence and strength of recommendations. BMJ. 2004;328(7454):1490-4.

10. Jiang ZM, Cao JD. The impact of micronized purified flavonoid fraction on the treatment of acute haemorrhoidal episodes. Curr Med Res Opin. 2006;22(6):1141-7.

\section{CONFLITO DE INTERESSES}

Os autores declaram não ter quaisquer conflitos de interesse.

\author{
ENDEREÇO PARA CORRESPONDÊNCIA \\ Filipe Fontes Alves \\ E-mail: f.fontes.alves@gmail.com \\ https://orcid.org/0000-0002-3392-9989
}

Recebido em 07-03-2019

Aceite para publicação em 20-08-2019

\section{ABSTRACT}

\section{FLAVONOIDS FOR SYMPTOMATIC HEMORRHOIDS: AN EVIDENCE-BASED REVIEW}

Objective: Hemorrhoids result from the swelling of the hemorrhoidal venous plexus and may result in symptoms such as pain, itching, mucous discharge, bleeding, and discomfort due to prolapse. One of the therapeutic options is oral flavonoids. Its effectiveness remains a controversial point of debate. As such it is our goal to determine, through an evidence-based review, whether the use of flavonoids for symptomatic control is a valid therapeutic option.

Data sources: National Guideline Clearinghouse, NICE Guidelines Finder, The Cochrane Library, DARE, Bandolier, MEDLINE, and bibliographic references of the selected articles were reviewed.

Review methods: Definition of the inclusion criteria through the PICO methodology: Population - individuals with symptomatic hemorrhoids; Intervention - Flavonoids; Comparison - Placebo; Outcome - Efficacy for symptomatic control. Survey of clinical guidelines, systematic reviews, and randomized clinical trials using the MeSH terms 'Hemorrhoids' and 'Flavonoids', published in the last 10 years, in Portuguese, Spanish, French, and English. The American Academy of Family Physician's Strength of Recommendation Taxonomy scale was applied to grade de evidence.

Results: The search indicated 13 articles, two of which fulfilled the inclusion criteria, with high scientific quality and from which the conclusions were drawn, all favorable to the use of flavonoids, but with variable benefit according to the symptom explored.

Conclusions: The available evidence suggests that there is a benefit in the use of flavonoids to control symptomatic hemorrhoids (versus placebo). Its use is recommended for the control of pain and bleeding (strength of recommendation A). It is necessary to consider limitations such as the subjectivity of symptom evaluation performed by patients; the influence of diet; flavonoids comprising a vast group of drugs; there being no standard dose for treatment. Additional studies are needed to clarify these issues.

Keywords: Hemorrhoids; Flavonoids. 